\title{
Analysis of Mechanical Behavior of Composite Tissues Using Vibrational Optical Coherence Tomography
}

\author{
Frederick H. Silver ${ }^{1}$, Ruchit G. Shah ${ }^{2}$ \\ ${ }^{1}$ Department of Pathology and Laboratory Medicine, Robert Wood Johnson Medical School, Rutgers, The State University of New \\ Jersey, Piscataway, NJ, USA \\ ${ }^{2}$ Graduate Program in Biomedical Engineering, Rutgers, The State University of New Jersey, Piscataway, NJ, USA \\ Email: fhsilver@hotmail.com
}

How to cite this paper: Silver, F.H. and Shah, R.G. (2017) Analysis of Mechanical Behavior of Composite Tissues Using Vibrational Optical Coherence Tomography. World Journal of Mechanics, 7, 271-282. https://doi.org/10.4236/wjm.2017.710022

Received: July 25, 2017

Accepted: October 17, 2017

Published: October 20, 2017

Copyright ( 2017 by authors and Scientific Research Publishing Inc. This work is licensed under the Creative Commons Attribution International License (CC BY 4.0).

http://creativecommons.org/licenses/by/4.0/ (c) (i) Open Access

\begin{abstract}
Extracellular matrices (ECMs) found in vertebrate tissues are fiber reinforced composite materials that prevent premature mechanical failure, store, transmit, and dissipate mechanical energy generated by the musculoskeletal system. We have developed a new method using optical cohesion tomography and vibrational analysis to non-destructively and non-invasively measure the mechanical properties of composite tissues and polymeric materials. In addition, this method can be used to measure the moduli of individual components of composite materials and perform "mechanical spectroscopy" on materials. In addition, we propose that measurement of the resonant frequency of a material minimizes the viscoelastic behavior of a composite material. This approach simplifies the analysis of mechanical behavior of polymers and others materials that demonstrate time-dependence to their properties.
\end{abstract}

\section{Keywords}

Collagen Fibers, Elastic Fibers, Dermis, Mechanical Properties, Optical Coherence Tomography, Vibrational Analysis, Skin, Cartilage, Bone, Composites

\section{Introduction}

Extracellular matrices (ECMs) are multi-component composite structures found in vertebrates that contain collagen and elastic fibers, proteoglycans, ions, water, growth factors, cell attachment factors and cells [1] [2] [3]. ECMs are found in 
cartilage, tendons, ligaments, skin, vessel walls, organ parenchyma and musculoskeletal tissues. Human skin is a multi-layered organ and can be separated into two distinct regions, the dermis and the epidermis [4].

The epidermis consists of several cell layers beginning with a layer of viable basal keratinocytes that differentiate into a cornified non-viable layer of squamous epithelium that covers the surface. The epidermis and lower dermis are separated by a flexible basement membrane on which cells of the epidermis rest [4]. The mechanical properties of skin and isolated dermis are similar suggesting that the epidermis normally does not play a significant role in mechanical properties of skin [2]. However, under extreme loading conditions the epidermis thickens and produces calluses that stiffen the skin.

The dermis is a composite material containing two parallel networks of collagen and elastic fibers. It can then be sub-divided into two layers, the superficial papillary and deep reticular dermis. The deep reticular layer, which represents about $75 \%$ of the skin by volume, serves as the essential macromolecular support structure imparting skin with its overall strength and elasticity [4]. Human skin is composed, on a wet weight percentage basis, of approximately $60 \%-72 \%$ water, $30 \%$ collagen, $0.2 \%$ elastin, and $0.03 \%-0.035 \%$ glycosaminoglycans, with cellular components and non-collagenous proteins also comprising a small fraction of the wet weight [5]. Collagen types I and III comprise approximately $70 \%$ to $80 \%$ of the dry weight of skin, and are the most important structural component of skin [4] [5]. The collagen meshwork present in the dermis provides the bulk of the skin support structure, and imparts it with the majority of its mechanical strength [6] [7] [8] [9] [10].

Elastic tissue in skin forms an independent three-dimensional network of branched fibers of variable diameter and elastin content, which spans from the papillary layer to the deep dermis. Mechanically, the elastic fiber network of skin is in parallel with that of collagen [11] and in skin from older individuals elastic fibers appear to fray and contain holes. Diameters of elastic fibers increase from about $1 \mu \mathrm{m}$ to $2 \mu \mathrm{m}$ in proceeding from the papillary to the reticular dermis. They form a continuous network that can be isolated by treatment with strong alkali and autoclaving after removal of other components. The relative volume of elastic fibers increases from about $0.7 \%$ to about $2.5 \%$ in proceeding from the papillary to the reticular dermis [4] [5]. The role of ECM in skin is to dissipate impact loads and resist failure while that of ECM in cartilage functions primarily to store and transmit excess energy while also resisting impact loads [11].

Hyaline cartilage is a composite ECM found on the ends of long bones. It is stretched in tension over the joint surface and contains cells termed chondrocytes that are up-regulated by increased loading [11]. Cartilage is composed of chondrons [12] which consist of cells, chondrocytes, and pericellular matrix. The pericellular matrix is enriched in hyaluronic acid and proteoglycans [13]. Chondrons in cartilage are embedded within a collagenous matrix composed of predominantly type II collagen along minor collagen types IX, and XI [14]. Type X 
collagen is found in the calcified zone in association with types II and XI collagens [11].

Cartilage is made up of several structural components that have been termed layers or zones. The collagen fibers in the superficial zone are primarily oriented along the surface, while those in the deep zone are oriented perpendicular to the subchondral bone (see [14] for a review). In the intermediate zone collagen fibrils are randomly oriented before they become parallel to the surface in the superficial zone. Mineralizing cartilage, found at the interface with the subchondral bone, contains collagen fibers that run parallel to axis of the subchondral bone and serves as the transition from soft spongy cartilage of the intermediate zone to hard rigid bone that supports loads in the joint. Thus cartilage is a composite material containing both mineralized and unmineralized collagen fibers that have been shown to possess different mechanical properties [14].

The major function of collagen fibers in skin and cartilage is to prevent tissue failure by withstanding deformation and dissipating energy during stretching [11]. Tensile testing of human skin and cartilage shows that these tissues possess the characteristic time-dependent properties of viscoelastic materials [8] [9] [14] [15] [16] [17]. The typical stress-strain curve for human skin presents as a biphasic response that is composed of linear low- and high-strain regions [8] [9] [14] [18] [19] [20]. In the low-strain or toe region, skin primarily behaves elastically and its behavior is thought to be dominated by elastic fibers which act to recover small strains in the collagen network [8] [9] [14] [18] [19] [20] [21] [22]. As the tissue is strained beyond the toe region, in the high-strain region, collagen fibrils become aligned and are recruited to absorb and store the strain energy [2] [19] [20]. The high-strain response includes a viscous component that is thought to be associated with energy dissipation of the applied load through viscous and molecular sliding of collagen fibrils in the extracellular matrix [2] [10]. Thus dermis contains two fiber networks that have different mechanical properties.

Several methods have been used to evaluate the mechanical properties of ECMs over the last 40 years including uniaxial and biaxial tensile testing, indentation and rotational tests [3] [4] [8] [9] [14] [15] [16] [17] [19] [20] [21] [22]. In addition, new tests including ultrasound elastography (UE), optical cohesion tomography (OCT), optical cohesion elastography (OCE), and vibrational analysis combined with OCT have been used to study the mechanical properties of tissues in health and disease. Most of these techniques require the assumptions that the material is linearly elastic, Poisson's ratio is close to 0.5 and that viscoelasticity does not dramatically affect the resulting properties. However, ECMs are non-linear materials that are viscoelastic and have upward curvature to the stress-strain curve. This fact makes determination of the stiffness very difficult since the tangent to the stress-strain curve is constantly changing [14]-[20]. However, despite all of these problems, there is a need to be able to characterize the mechanical properties of ECMs since this would give clinicians valuable information concerning the me- 
chanical properties of skin with respect to location and the directions of Langer's lines. In addition, changes associated with tumor formation, wound healing, scarring and the efficacy of cosmetic and surgical treatments could be evaluated quantitatively.

During the last three decades methodology has been developed to estimate the viscoelastic mechanical properties of skin [20] and decellularized dermis [2] [11] [14]. Our results suggest that the non-linearity of the stress-strain curves can be analyzed by dividing the stress into elastic and viscous components [2] [11] [14] [20]. The elastic component can be measured as the stress at equilibrium in an incremental stress-strain experiment [2] [11] [14] [20]. This test is characterized by the sequential addition of loading increments followed by a relaxation step between each loading step [2] [11] [14] [20]. When all the equilibrium stresses are plotted versus the strain, an elastic stress-strain curve is obtained. Equilibrium stress-strain curves can then be used to calculate the elastic modulus as the tangent to the curve [2] [11] [14] [20].

The elastic modulus of a number of collagenous tissues has been shown to be independent of strain-rate for all strain rates up to $10,000 \%$ per minute [11]. If the slope of the elastic stress-strain curve at high strains is divided by the collagen content of the tissue, the fraction of collagen aligned with the tensile direction, and the change in strain after the collagen fibers of the tissue are stretched in tension, the resultant number is between 4.0 and $7.0 \mathrm{GPa}$, values similar to those reported for the stiffness of the collagen molecule [11]. The slope of the viscous stress-strain curve reflects the length of the collagen fibrils and the viscous sliding of collagen fibrils by each other during tensile deformation [11].

While dermis and cartilage have been shown to contain several different elements that have different mechanical properties [2] [11], until recently there was no method that could be used to evaluate the mechanical properties of individual components of a composite material non-invasively and non-destructively [21] [22]. The introduction of optical cohesion tomography in combination with vibrational analysis has paved the way to do "mechanical spectroscopy" on tissues without having to test the individual layers of the materials separately [22]. The purpose of the paper is to review data on dermis and cartilage to underscore the use of this technique for analysis of the mechanical properties of composite materials non-invasively and non-destructively.

\section{Methods}

\subsection{Sample Preparation}

Human decellularized dermis was obtained from allograft tissue as described previously [2] [21] [22] [23] (see Table 1). Decellularized human dermal samples were tested after immersion in phosphate buffer solution as described elsewhere [21] [22]. All samples were tested wet after soaking in phosphate buffer solution at $\mathrm{pH} 7.4$ for at least 30 minutes. Processing and testing steps were conducted at $22^{\circ} \mathrm{C}$. 
Table 1. Moduli values for ECM components measured using OCT and vibrational analysis. Note the strain is the external strain and does not account for any internal strain such is present in femoral cartilage-subchondral bone composites.

\begin{tabular}{|c|c|c|c|c|}
\hline Sample & Resonant Frequency $(\mathrm{Hz})$ & Vibrational Testing Modulus ( $\mathrm{MPa}$ ) & Strain $(\%)$ & Thickness (mm) \\
\hline \multirow{3}{*}{ Decellularized Dermis } & $153.33 \pm 5.77$ & $2.57 \pm 0.2$ & 5 & \multirow{3}{*}{1.07} \\
\hline & $246.67 \pm 5.77$ & $6.65 \pm 0.31$ & 14 & \\
\hline & $346.67 \pm 5.77$ & $13.14 \pm 0.44$ & 20 & \\
\hline \multirow[b]{2}{*}{ Pig Skin } & $93.33 \pm 5.77$ & $0.77 \pm 0.094$ & \multirow[b]{2}{*}{5} & \multirow[b]{2}{*}{3.05} \\
\hline & $203.33 \pm 5.77$ & $3.61 \pm 0.2$ & & \\
\hline \multirow{3}{*}{ Bovine Cartilage } & $246.67 \pm 5.77$ & $4.96 \pm 0.23$ & \multirow{3}{*}{2} & \multirow{3}{*}{2.08} \\
\hline & $550 \pm 10$ & $24.65 \pm 0.53$ & & \\
\hline & $663.33 \pm 5.77$ & $35.03 \pm 0.61$ & & \\
\hline Subchondral Bone & $620 \pm 10$ & $31.92 \pm 1.03$ & 2 & 1.39 \\
\hline
\end{tabular}

Depilated pig skin, with a thickness of approximately $3 \mathrm{~mm}$, composed of epidermis and dermis, was obtained at slaughter from Spear Products (Coopersburg, PA) and stored at $4^{\circ} \mathrm{C}$. All samples were tested wet after soaking in phosphate buffer solution at $\mathrm{pH} 7.4$ for at least 30 minutes. Processing and sample testing steps were conducted at $22^{\circ} \mathrm{C}$ as described previously [21] [22].

Bovine femoral cartilage with attached subchondral bone with a thickness of approximately $2 \mathrm{~mm}$ was obtained from Spear Products (Coopersburg, PA). It was stored at $-40^{\circ} \mathrm{C}$ until it was tested. It was thawed before use and soaked in phosphate buffer at $\mathrm{pH} 7.4$ for at least 30 minutes before testing at $22^{\circ} \mathrm{C}$. Cartilage was removed from the subchondral bone by mechanical scraping using a surgical blade to evaluate the mechanical properties of the bone alone after testing both bone and cartilage together.

\subsection{Mechanical Testing}

\subsubsection{OCT and Vibrational Analysis in Vitro}

Transverse forces were applied to the sample by positioning an acoustic loudspeaker (Intervox S225RA-40) beneath the sample. A function generator (Agilent) was used to drive the speaker with sinusoidal waveforms at varying amplitude and frequency as discussed previously [21] [22]. Varying axial deformations of $1 \%-15 \%$ were applied through adjustment of the graduated translation stage.

Transverse sample displacement was measured by spectral-domain optical coherence tomography (SD-OCT), a non-contact, interferometric technique as discussed previously [21] [22]. The SD-OCT system uses a fiber-coupled superluminescent diode light source with $1325 \mathrm{~nm}$ center wavelength and $100 \mathrm{~nm}$ bandwidth (full-width at half maximum) as described previously [21] [22].

The resonant frequency of each sample was initially estimated by measuring the transverse displacement resulting from sinusoidal driving frequencies ranging from $50 \mathrm{~Hz}$ to $1000 \mathrm{~Hz}$, in steps of $50 \mathrm{~Hz}$. Once the region where the maximum frequency was identified, smaller steps of $10 \mathrm{~Hz}$ were used to more accu- 
rately identify the peak frequency and the actual resonant frequency, $f_{n}$.

$$
E=m\left(2 \pi f_{n}\right)^{2}\left(\frac{L}{A}\right)
$$

The modulus from vibrational studies, $E$, was determined using Equation (1) where $m, L$ and $A$ are the sample mass, length and cross-sectional area.

\subsubsection{Calibration Studies}

A variety of samples made from silicone rubber, decellularized dermis, and chemically modified decellularized dermis were tested in uniaxial tension and using vibrational analysis to establish a calibration curve between the moduli calculated from tensile measurements and those derived from vibrational measurements in vitro. These results have been published elsewhere [22].

The relationship between the modulus measured using vibrational $\left(E_{V}\right)$ and tensile $(E t)$ measurements was reported to be approximately linear and the equation of the line was found to be:

$$
E V=1.026 E t+0.0046
$$

where, $E_{V}$ and $E t$ are the moduli measured in MPas. The correlation coefficient between these moduli is 0.984 as previously reported [22]. The relationship between tensile and vibrational moduli was approximated using Equation (2). The material behavior was reported to be reversible for strains less than about $14 \%$ for up to three cycles of tensile testing [21] [22]. The relationship between moduli determined from vibrational and tensile experiments has been reported earlier [21] [22]. Using the vibrational method, the modulus of a material at a specific strain can be calculated.

The resonant frequency and moduli were determined for human decellularized skin, pig skin, intact bovine cartilage, and subchondral bone as shown in Table 1. Figure 1 illustrates that the modulus determined from vibrational experiments is dependent on strain. Plots of weighted displacement versus frequency are shown for decellularized human dermis (Figure 2), pig skin (Figure 3), intact bovine femoral cartilage (Figure 4) and subchondral femoral bovine bone (Figure 5). The resonant frequency varied from about $100 \mathrm{~Hz}$ over $600 \mathrm{~Hz}$ for subchondral bone (Table 1). The modulus determined from vibrational measurements varied from about $2.57 \mathrm{MPa}$ at a strain of $5 \%$ for decellularized human skin to over $30 \mathrm{MPa}$ for subchondral bone. Figure 1 and Figure 2 show that the resonant frequency for decellularized dermis is dependent on the applied strain, while Figure 3 illustrates that pigskin demonstrates two resonant frequencies one at 90 $\mathrm{Hz}$ and another at $200 \mathrm{~Hz}$ at a strain of 5\%. Finally, Figure 4, illustrates that bovine femoral cartilage shows three resonant frequencies: one at $250 \mathrm{~Hz}$ one at 550 $\mathrm{Hz}$ and the final one at $660 \mathrm{~Hz}$. After the cartilage is removed from the subchondral bone with a scalpel, the resonant frequency of subchondral bone becomes 620 $\mathrm{Hz}$ (Figure 5). 


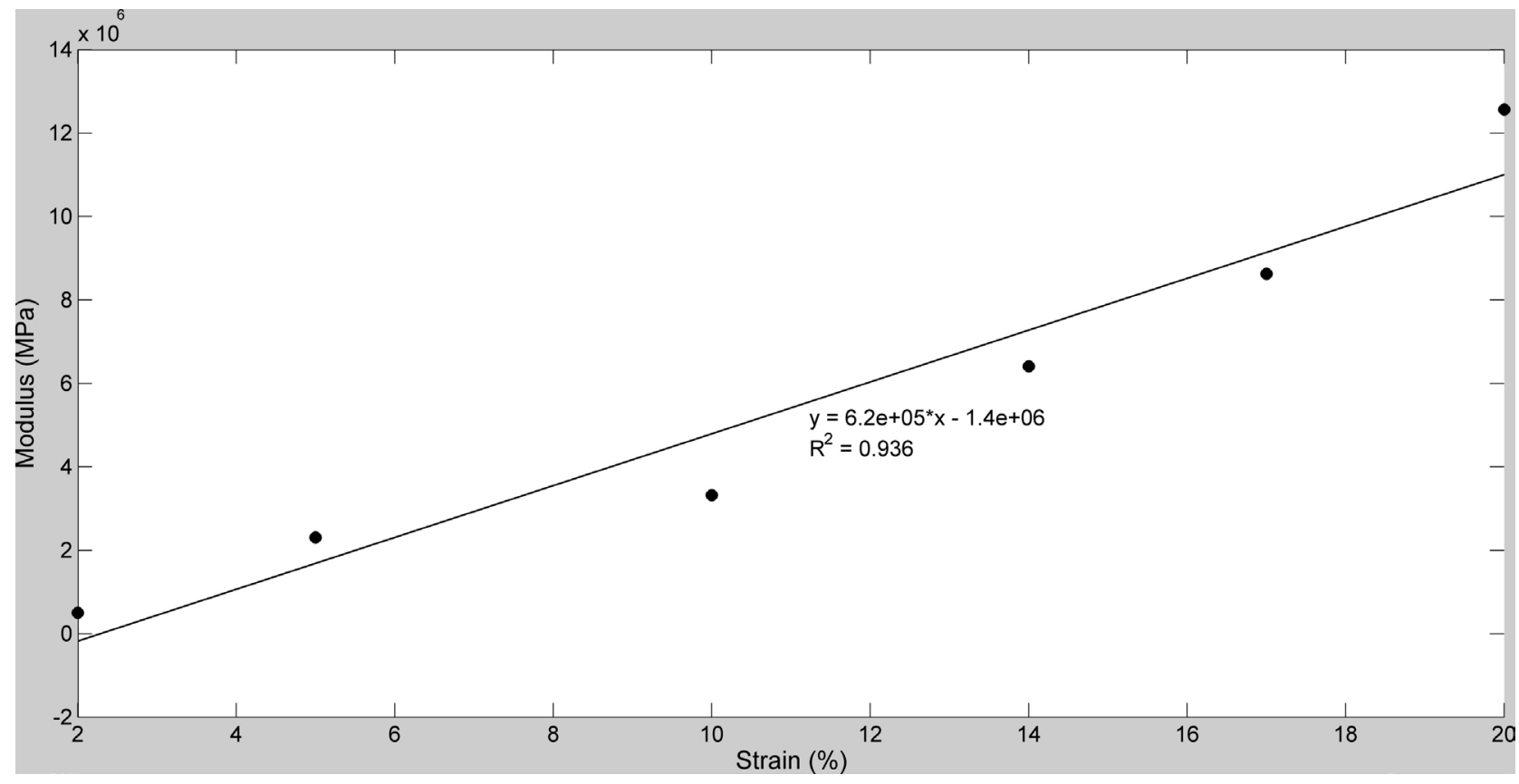

Figure 1. Plot of modulus determined from OCT and vibrational studies versus strain for decellularized dermis. Note the resonant frequency and modulus increases with strain.

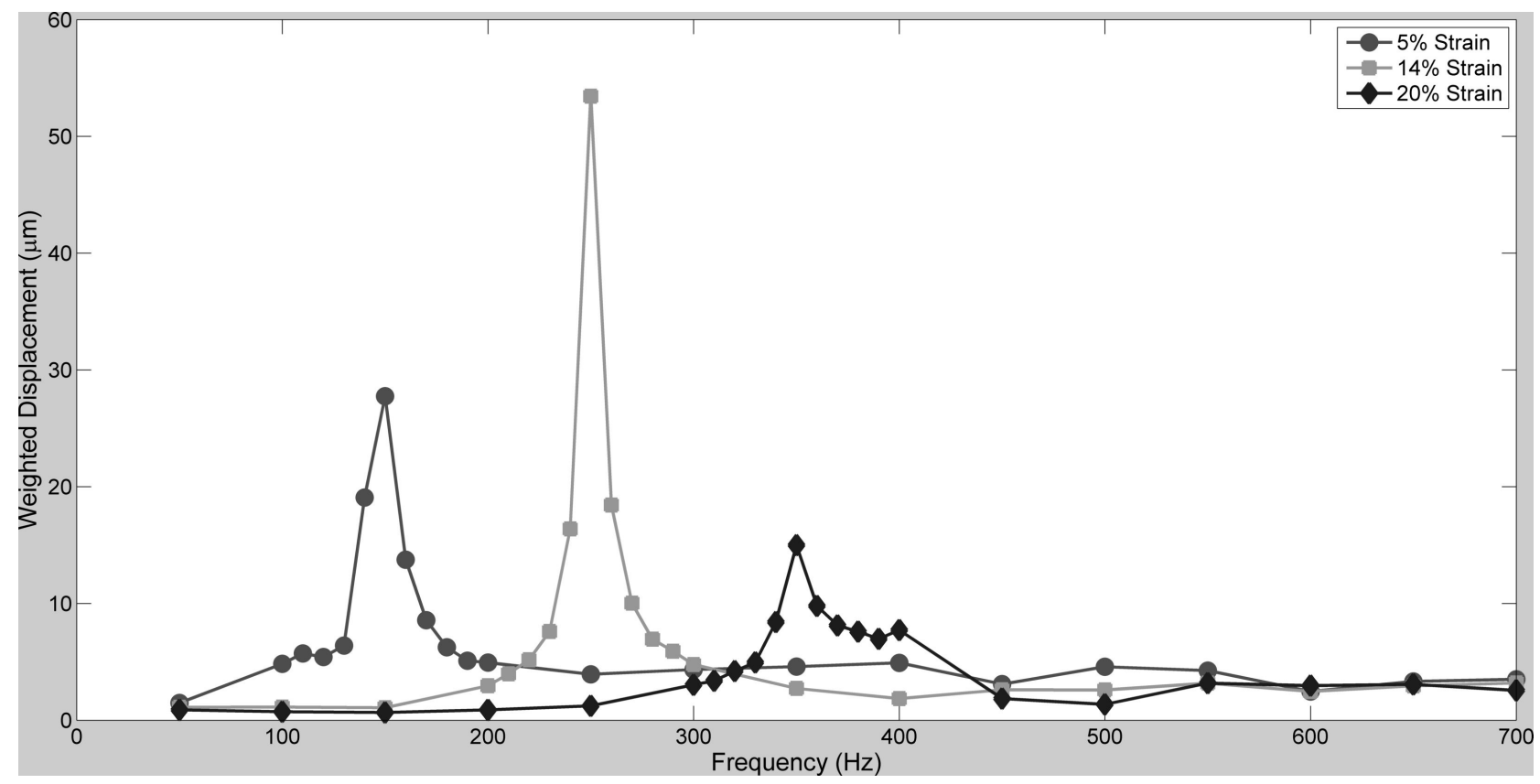

Figure 2. Weighted displacement versus frequency for decellularized dermis at $5 \%, 14 \%$ and $20 \%$ strain. The resonant frequencies measured were $150 \mathrm{~Hz}$ (5\% strain), $250 \mathrm{~Hz}$ (14\% strain), and $350 \mathrm{~Hz}$ (30\% strain) as listed in Table 1.

\section{Discussion}

The ability of researchers and scientists to understand the relationship between the hierarchical structure of composite materials and their mechanical properties is important to design new materials that can be used as implants as well as new composite materials that can be used in industrial applications. While the mechanical 


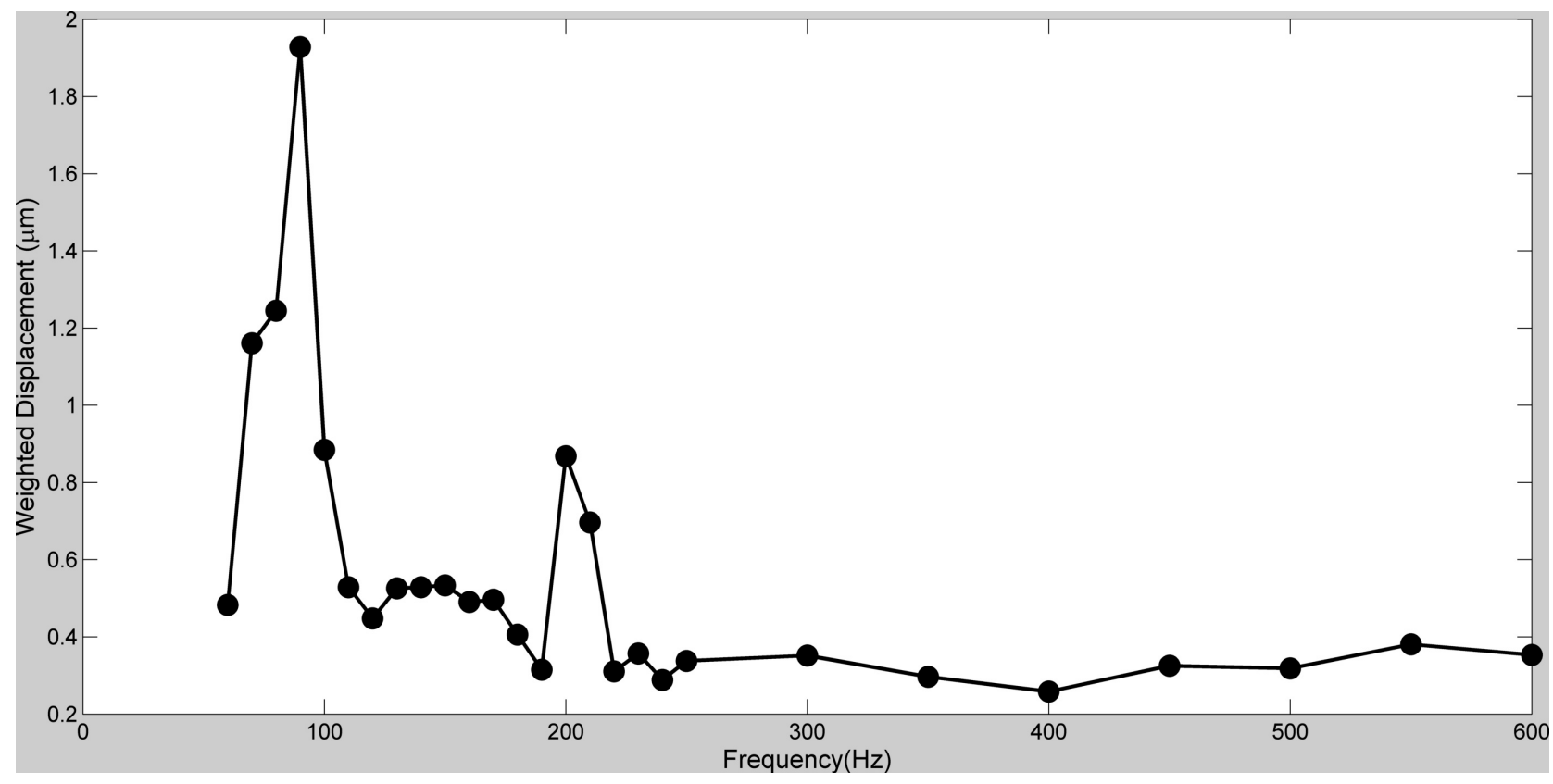

Figure 3. Weighted displacement versus frequency for pig skin stretched to a strain of $5 \%$. The resonant frequencies of the pig skin were $90 \mathrm{~Hz}$ and $200 \mathrm{~Hz}$ as shown in Table 1 .

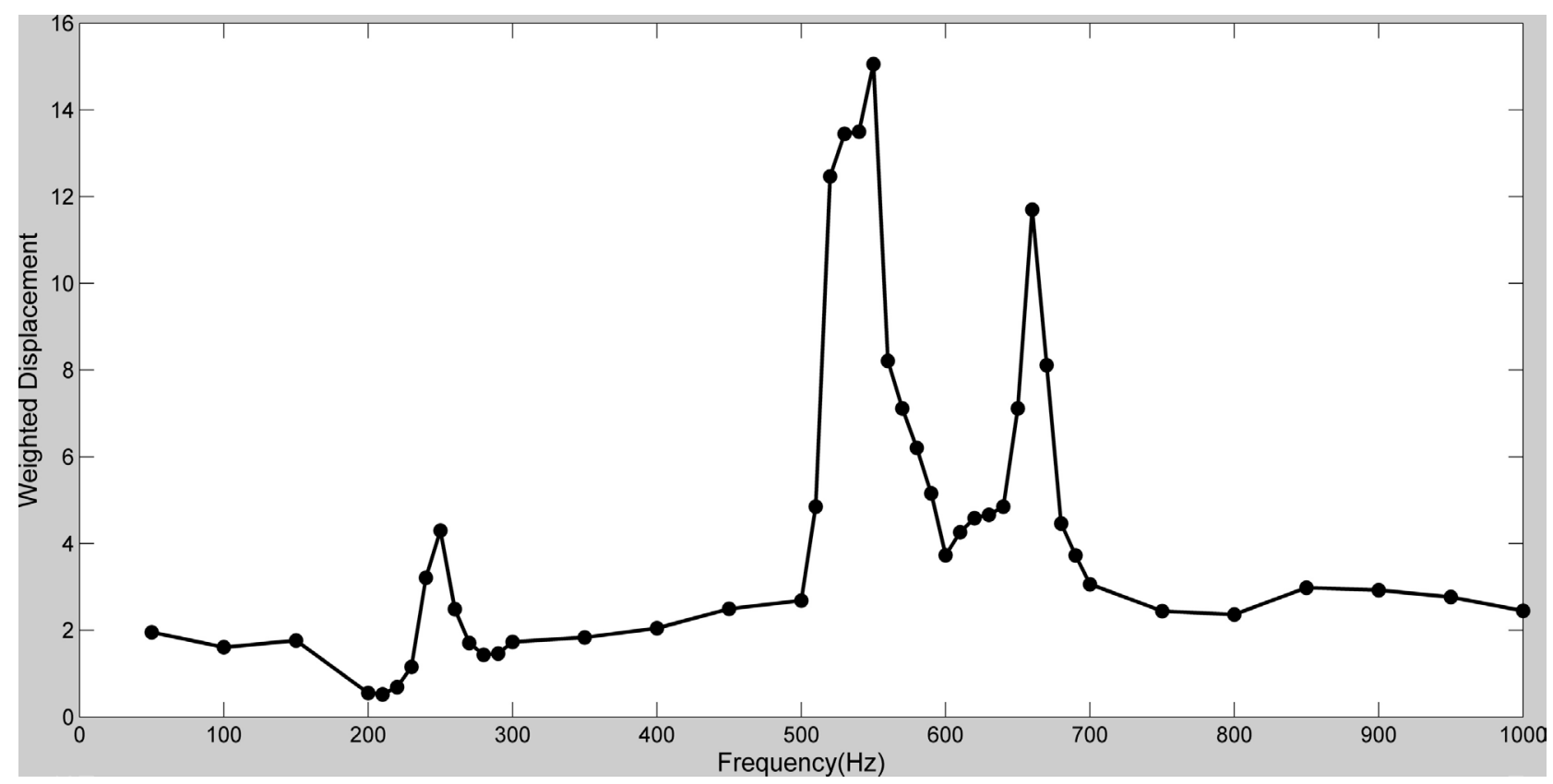

Figure 4. Weighted displacement versus frequency for bovine femoral cartilage with a layer of subchondral bone. The resonant frequencies observed were $250 \mathrm{~Hz}, 550 \mathrm{~Hz}$ and $660 \mathrm{~Hz}$ as is listed in Table 1 .

properties of implants and industrial composites are complex; much progress has been made in understanding the relationship between structure and mechanical properties and how to facilitate analysis of viscoelastic materials.

We recently reported a correlation between modulus values calculated from the slopes of incremental tensile stress-strain curves and measurements based on natural frequency determination for both tissues and silicone rubber samples 


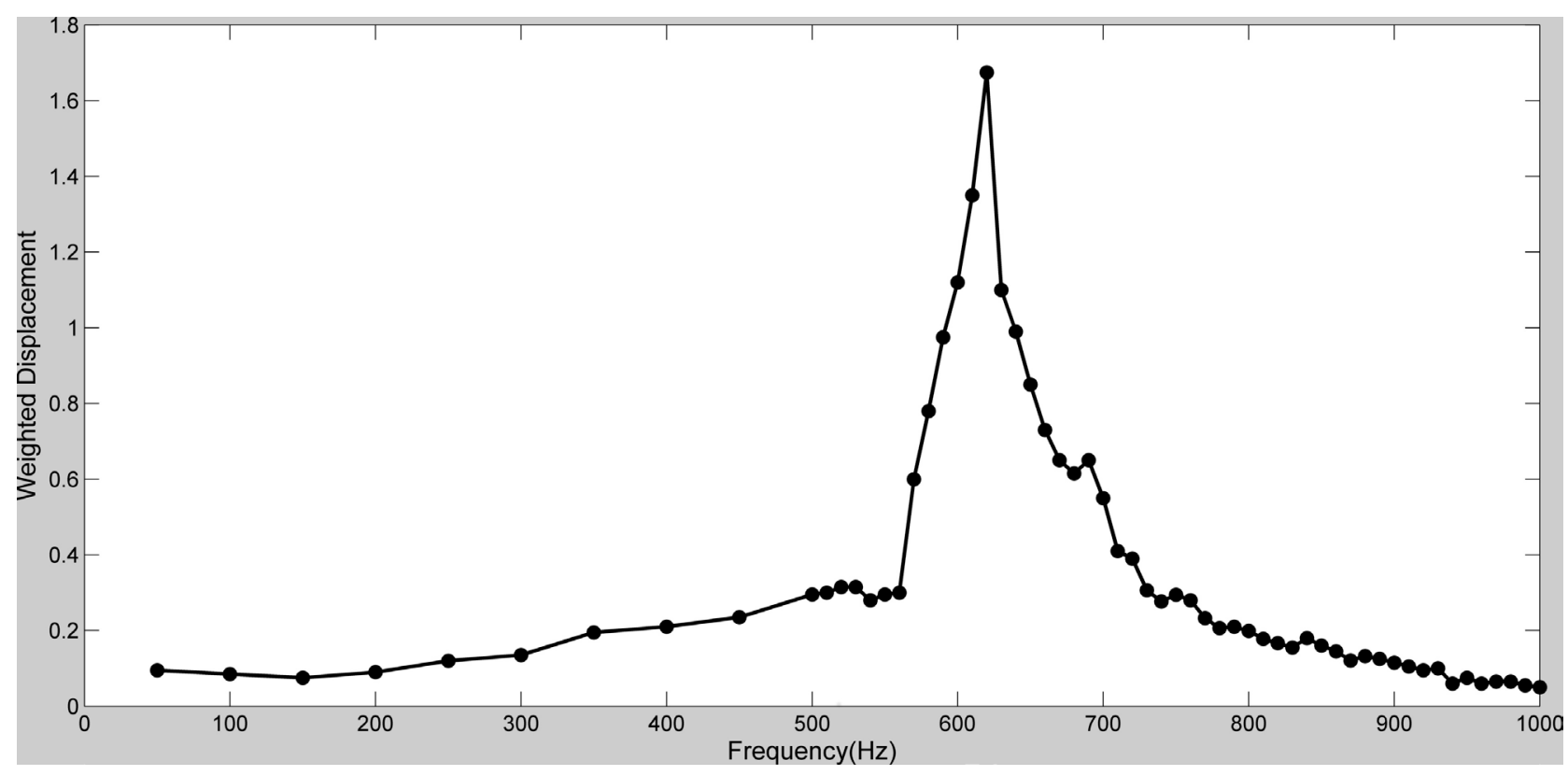

Figure 5. Weighted displacement versus frequency for bovine femoral cartilage and subchondral bone after most of the cartilage was removed with a scalpel. The resonant frequency for subchondral bone is $620 \mathrm{~Hz}$ as indicated in Table 1.

[21] [22]. These results suggest that the modulus determined from conventional methods (tensile stress-strain measurements) is consistent with the modulus values calculated from measurement of the natural frequency determined from a combination of OCT and vibrational analysis [21] [22]. The use of Equation (2) to construct a calibration curve between modulus and natural frequency measurements results in a high correlation coefficient between these two independent measurements without assuming a value of Poisson's ratio. This suggests that use of vibrational analysis in conjunction with OCT gives modulus values that are similar to those found from uniaxial tensile measurements, a "gold standard" method that has been used to report stress-strain behavior of materials.

Unlike many other materials, ECMs have a stress-strain relationship that curves upward with increasing strain [2] [11] [14] [20]. This indicates that as the collagen fibers are aligned and stretched along the tensile axis, the material stiffens since more collagen fibers bear the load [2] [11] [14] [20].

Using vibrational analysis, both natural and synthetic materials can be characterized non-invasively. When a material contains two or more major components more than one vibrational peak is observed with unique resonant frequencies. It is then necessary to characterize the origin of each of these peaks in the vibrational spectrum.

In the case of ECMs this task is simplified by analyzing the behavior of decellularized human dermis since it contains a single component, e.g. collagen fibers [2]. The modulus of collagen fibers in skin increases from about $2.57 \mathrm{MPa}(5 \%$ strain) to $13.36 \mathrm{MPa}$ at a strain of $20 \%$ as the strain is increased [22]. The resonant frequency and calculated modulus are therefore dependent on the tissue strain and the composition. If one next examines the weighted displacement curve 
versus frequency for pig skin we see two peaks; one at $90 \mathrm{~Hz}$ and another at 200 $\mathrm{Hz}$ at a strain of $5 \%$ (Figure 2). The peak at $200 \mathrm{~Hz}$ appears to be the collagen peak since it has a modulus of about $3.55 \mathrm{MPa}$ as opposed to the one for collagen in decellularized dermis with a modulus of $2.57 \mathrm{MPa}$. The peak at $90 \mathrm{~Hz}$ is a peak due to elastic tissue since it has a modulus of $0.75 \mathrm{MPa}$ very close to that reported for elastic tissue in previous publications [2] [22].

The data obtained with bovine femoral cartilage suggests that the collagen peak at $250 \mathrm{~Hz}$ may reflect the collagen fibers in the superficial and intermediate zones. They have a modulus of $5.09 \mathrm{MPa}$ at an external strain of $2 \%$, while the peak at about $600 \mathrm{~Hz}$ appears to reflect the behavior of the subchondral bone on which the cartilage sits since it has a modulus of over $30 \mathrm{MPa}$ (Figure 4). The peak at 550 may reflect the transition zone between calcified cartilage and the subchondral bone. It is important to note that cartilage is stretched over the subchondral bone as previously reported [14] so the net strain would be $2 \%$, the external strain, and $9 \%$ (the estimated internal strain based on Figure 1) yielding a total strain of about $11 \%$. This is consistent with previous studies that suggest that when the cartilage is removed from the bone it curls up (see [14] for an explanation) suggesting that the residual strain in the material is significant [15].

The ability to measure the resonant frequency and modulus of individual components of composite materials is very desirable. It allows workers to design better implants and other fiber reinforced composite materials as well as to analyze for the presence of cracks non-invasively. The recent finding that the modulus measured using vibrational analysis and OCT at the resonant frequency is within $3 \%$ to $4 \%$ of the elastic modulus indicates that the viscoelasticity of composites is minimized using this technique [24]. This simplifies the analysis of the properties of viscoelastic composites since no correction for viscous loss is necessary and the modulus obtained is a "materials constant" at a particular strain.

\section{References}

[1] Burgeson, P. and Nimni, M.E. (1992) Collagen Types: Molecular Structure and Tissue Distribution. Clinical Orthopaedics and Related Research, 282, 250-257. https://doi.org/10.1097/00003086-199209000-00033

[2] Silver, F.H., Freeman, J. and DeVore, D. (2001) Viscoelastic Properties of Human Skin and Processed Dermis. Skin Research and Technology, 7, 18-23. https://doi.org/10.1034/j.1600-0846.2001.007001018.x

[3] Parry, D.A. and Craig, A.S. (1984) Growth and Development of Collagen Fibrils in Connective Tissue. In: Ruggeri, A. and Motta, P.M., Eds., The Ultrastructure of the Connective Tissue Matrix, Martinus Nijhoff Publishers, Boston, MA, 34-64. https://doi.org/10.1007/978-1-4613-2831-5_2

[4] Millington, P.F. and Wilkinson, R. (1983) Skin. Cambridge University Press, New York.

[5] Vitallaro-Zuccarello, L., Cappalletti, S., Rossi, V.P.D. and Sari-Gorla, M. (1994) Stereological Analysis of Collagen and Elastic Fibers in the Normal Human Dermis: Variability with Age, Sex, and Body Region. The Anatomical Record, 238, 153-162. https://doi.org/10.1002/ar.1092380202 
[6] Trelstad, R.L., Birk, D.E. and Silver, F.H. (1982) Collagen Fibrillogenesis in Tissues, in a Solution and from Modeling: A Synthesis. Journal of Investigative Dermatolo$g y$, 79, 109s-112s. https://doi.org/10.1111/1523-1747.ep12545945

[7] Uitto, J., Olsen, D.R. and Fazio, M.J. (1989) Extracellular Matrix of the Skin: 50 Years of Progress. Journal of Investigative Dermatology, 92, 61-77. https://doi.org/10.1038/jid.1989.34

[8] Oxlund, H., Maschot, J. and Vidiik, A. (1988) The Role of Elastin in the Mechanical Properties of Skin. Journal of Biomechanics, 21, 213-218. https://doi.org/10.1016/0021-9290(88)90172-8

[9] Gosline, J., Lillie, M., Carrington, E., Guerette, P., Ortlepp, C. and Savage, K. (2002) Elastic Proteins: Biological Roles and Mechanical Properties. Philosophical Transactions of the Royal Society London, 357, 121-132. https://doi.org/10.1098/rstb.2001.1022

[10] Silver, F.H., Horvath, I. and Foran, D.J. (2002) Mechanical Implications of the Domain Structure of Fiber-Forming Collagens: Comparison of the Molecular and Fibrillar Flexibilities of the Alpha1-Chains Found in Types I-III Collagen. Journal of Theoretical Biology, 216, 243-54. https://doi.org/10.1006/jtbi.2002.2542

[11] Silver, F.H. (2006) Mechanosensing and Mechanochemical Transduction in Extracellular Matrix. Springer, New York.

[12] Poole, C.A., Flint, M.H. and Beaumont, B.W. (1987) Chondrons in Cartilage: Ultrastructural Analysis of the Pericellular Microenvironment in Adult Human Articular Cartilage. Journal of Orthopaedic Research, 5, 509-522. https://doi.org/10.1002/jor.1100050406

[13] Poole, C.A., Flint, M.H. and Beaumont, B.W. (1984) Morphological and Functional Interrelationships of Articular Cartilage Matrices. Journal of Anatomy, 138, 113-138.

[14] Silver, F.H. and Bradica, G. (2002) Mechanobiology of Cartilage: How Do Internal and External Stresses Affect Mechanochemical Transduction and Elastic Energy Storage? Biomechanics and Modeling in Mechanobiology, 1, 219-238. https://doi.org/10.1007/s10237-002-0017-9

[15] Fung, Y.C. (1993) Biomechanics Mechanical Properties of Living Tissue. Springer-Verlag, New York.

[16] Yamada, H. (1970) Strength of Biological Materials. Williams and Wilkins, Baltimore.

[17] Silver, F.H., Kato, Y.P., Ohno, M. and Wasserman, A.J. (1992) Analysis of Mammalian Connective Tissue: Relationship between Hierarchical Structures and Mechanical Properties. Journal of Long-Term Effects of Medical Implants, 2, 165-198.

[18] Elsner, P., Wilhelm, K.P., Maibach, H.I. and Berardesca, E. (2001) Bioengineering of the Skin: Skin Biomechanics. CRC Press, New York.

[19] Vogel, H.G. (1987) Age Dependence of Mechanical and Biochemical Properties of Human Skin. Part I: Stress-Strain Experiments, Skin Thickness and Biochemical Analysis. Bioengineering and the Skin, 3, 67-91.

[20] Dunn, M.G. and Silver, F.H. (1983) Viscoelastic Behavior of Human Connective Tissues: Relative Contribution of Viscous and Elastic Components. Connective Tissue Research, 12, 59-70. https://doi.org/10.3109/03008208309005612

[21] Shah, R., DeVore, D., Pierce, M.G. and Silver, F.H. (2016) Morphomechanics of Dermis-A Method for Non-Destructive Testing of Collagenous Tissues. Skin Research and Technology. 
[22] Shah, R., Pierce, M.C. and Silver, F.H. (2017) A Method for Non-Destructive Mechanical Testing of Tissues and Implants. Journal of Biomedical Materials Research Part $A, 105,5-22$.

[23] Seehra, G.P. and Silver, F.H. (2006) Viscoelastic Properties of Acid- and Alkaline-Treated Human Dermis: A Correlation between Total Surface Charge and Elastic Modulus. Skin Research and Technology, 12, 190-198.

https://doi.org/10.1111/j.0909-752X.2006.00150.x

[24] Shah, R.G. and Silver, F.H. (2017) Viscoelastic Behavior of Tissues and Implant Materials: Estimation of the Elastic Modulus and Viscous Contribution using Optical Coherence Tomography and Vibrational Analysis. Journal of Biomedical Technology and Research, 3, 105-109. 